\title{
Determination of strain and damage profiles in irradiated materials: application to cubic zirconia irradiated at high temperature
}

\author{
J. Channagiri ${ }^{1}$, A. Boulle ${ }^{1}$, A. Debelle ${ }^{2}$ \\ ${ }^{1}$ Science des Procédés Céramiques et Traitements de Surface, CNRS UMR 7315, Centre \\ Européen de la Céramique, 12 rue atlantis, 87068 Limoges Cedex, France \\ ${ }^{2}$ Centre de Spectrométrie Nucléaire et Spectrométrie de Masse, Université Paris-Sud, CNRS- \\ IN2P3, 91405 Orsay Cedex, France
}

\begin{abstract}
A methodology is presented that allows to retrieve strain and damage profiles in irradiated single crystals. The approach makes use of high-resolution X-ray diffraction $\theta-2 \theta$ scans coupled with numerical simulations of the diffraction profiles. The potential of the method is illustrated with Cubic yttria-stabilized zirconia single crystals, irradiated with $4 \mathrm{MeV} \mathrm{Au}^{2+}$ ions at different temperatures $\left(25,500\right.$ and $\left.800^{\circ} \mathrm{C}\right)$. The simulations reveal that upon increasing ion fluence, the width of the damaged region increases and both the strain and damage levels inside this region increase. The damage build-up occurs according to a two-step mechanism: in the first step, the damage increases slowly up to a critical fluence, above which the second step takes place and is characterized by dramatic increase of the damage. The transition fluence is shifted towards lower values at higher temperatures.
\end{abstract}




\section{Introduction}

The stabilization and immobilization of highly radioactive nuclear wastes in a solid form is one of the most concerning issues facing the nuclear energy research [1]. Out of the available materials, cubic yttria-stabilized zirconia (YSZ) is foreseen as a material which could be used as an inert matrix for the transmutation of radiotoxic actinides [2]. Additionally, the cubic phase of YSZ is of particular interest because it is isostructural with radioactive fuel materials such as urania $\left(\mathrm{UO}_{2}\right)$, plutonia $\left(\mathrm{PuO}_{2}\right)$ and thoria $\left(\mathrm{ThO}_{2}\right)$, while being much easier to synthesize and to study than these radioactive counterparts. The understanding of the behavior of YSZ under various irradiation conditions is therefore of utmost importance in order to validate its use in the electronuclear industry.

In this context, ion irradiation performed in various conditions (high temperature for instance), is used as a tool to simulate, in a controlled way, the different sources of irradiations to which these materials are exposed to. From a structural point of view, ion irradiation mainly induces inhomogeneous atomic displacements in the subsurface region of the target material: these displacements may arise either from the ballistic collisions with the incident ions, or result from the strain fields associated to structural defects (vacancies, cluster of vacancies, dislocation loops, etc.) formed as a consequence of these collisions. Recently, it was demonstrated that the damage build-up in YSZ irradiated at room temperature (RT) and high temperature with a few $\mathrm{MeV}$ heavy ions occurs in a multistep process $[3,4,5]$. The transitions from one step to the next are triggered by the relaxation of the energy associated with a given defect structure [6]. From an experimental standpoint each step can be uniquely characterized by features such as the level of strain and damage and the nature of the radiation defects [7].

In this work, we pursue our study initiated in [5], on YSZ single crystals irradiated with $4 \mathrm{MeV}$ $\mathrm{Au}^{2+}$ ions at room and high temperatures $\left(500\right.$ and $\left.800^{\circ} \mathrm{C}\right)$, and we focus on the determination of the strain and damage depth profiles. The determination of these profiles from XRD data is hindered by the so called 'phase problem': atomic displacements affect the phase of the diffracted amplitude $E$, whereas in an actual XRD experiment only the intensity $E E^{*}$ can be measured, so that the phase (hence the information regarding atomic displacements) is partly lost in the diffraction experiment. Here we show that this problem can be circumvented by making use of a specifically developed approach [8] that mainly relies on fitting the XRD intensities with a versatile diffraction model and an efficient fitting algorithm. Experimental details regarding ion irradiation and characterization techniques are provided in section 2, while section 3 is devoted to describe the methodology of the approach used in this work. Finally, in section 4, the results obtained in the case of irradiated YSZ single crystals will be presented and discussed in details. Particular emphasis will be laid on the physical interpretation of the phenomenological parameters entering the model.

\section{Experimental}

The samples used in this study are cubic $\{100\}$-oriented zirconia single crystals, fully stabilized with $9.5 \mathrm{~mol} \% \mathrm{Y}_{2} \mathrm{O}_{3}$ (Crystal $\mathrm{GmbH}$, Berlin). YSZ crystals have the fluorite structure $(F m 3 m)$ with a bulk lattice parameter $\mathrm{a}_{\mathrm{YsZ}}=0.5145 \mathrm{~nm}$ and a density $\rho=5.9 \mathrm{~g} \mathrm{~cm}^{-3}$. The YSZ crystals were irradiated at high temperatures with $4 \mathrm{MeV} \mathrm{Au}^{2+}$ ions using the ARAMIS accelerator of the CSNSM in Orsay. The irradiations were performed at fluences ranging from $10^{13}$ to $10^{15} \mathrm{~cm}^{-2}$. Irradiations were performed $7^{\circ}$ off the normal of the surface crystals to avoid channeling 
phenomenon. The ion current was always kept lower than $0.1 \mu \mathrm{A} \mathrm{cm}{ }^{-2}$ in order to minimize target heating during irradiation.

The XRD experiments have been conducted on a PANalytical X'Pert PRO MRD diffractometer equipped with a standard $\mathrm{Cu}$ tube. An intense and monochromatic beam was obtained by using a multilayer mirror coupled with a four-reflection $\mathrm{Ge}(220)$ monochromator in asymmetric configuration; the resulting primary-beam divergence was $\sim 18 \operatorname{arcsec}\left(0.005^{\circ}\right)$. A three-bounce $\mathrm{Ge}(220)$ analyzer crystal was used to further limit the detector acceptance. Symmetric $\theta-2 \theta$ scans were recorded in the vicinity of the (400) Bragg reflection, i.e. around $2 \theta=73.575^{\circ}$. All measurements have been carried out at room temperature.

\section{Methodology}

Although the diffraction of X-rays from crystalline materials is known for a century [9], it still remains a topic of active research for the characterization of materials. Its most appealing features are its non-destructive and highly quantitative character. Moreover, since large X-ray beams are used (several mm wide) the results obtained are statistically representative of the whole sample.

Since the diffraction phenomenon arises from the interference of the X-ray waves scattered by individual atoms, X-ray diffraction is highly sensitive to perturbations affecting the positions of atoms. It is therefore especially relevant for the determination of lattice strain, random atomic displacements, etc. However, as mentioned in the introduction, atomic displacements affect the phase of the diffracted amplitude, whereas the quantity measured in practice is the intensity. As a consequence the atomic displacement profiles $u(z)$ (where $z$ is depth below the surface of the crystal) caused by ion irradiation cannot be straightforwardly obtained from the observed XRD data [10]. This issue can be circumvented by a fitting approach: (i) in a first step, generate a possible $u(z)$; (ii) then compute the corresponding intensity and compare it with the observed data; and (iii) refine step (i) until a perfect agreement is reached. This last step is most efficiently carried out using an automated fitting approach. We here briefly recall the principles underlying each step of the approach.

The effects of atomic displacements can be phenomenologically divided into two categories: nonrandom displacements, which correspond to lattice strains $e(z)$, and random displacements which can be characterized by the root-mean-squared atomic displacements $\left\langle u(z)^{2}\right\rangle^{1 / 2}$. Lattice strain is written as:

$$
e(z)=\frac{a(z)-a_{Y S Z}}{a_{Y S Z}}
$$

where $a(z)$ is the lattice parameter at depth $z$ and $a_{Y S Z}$ is the lattice parameter of virgin YSZ. Lattice strains correspond to a change in the lattice parameters and therefore only give rise to a shift of the diffraction peaks emanating from the strained region. Conversely, random atomic displacements affect the diffracted Bragg intensity: the diffracted amplitude is lowered by the socalled static Debye-Waller (DW) factor, which results from the fact that the structure factor of the material has to be averaged over all possible atomic displacements in the material. This average is often performed by assuming a Gaussian distribution of displacements (which is a consequence of the central limit theorem, stating that the sum of identically distributed random variables with finite variance converges to a Gaussian distribution). In such a case, the DW factor is simply written [11]: 
$D W(z)=\exp \left(-\frac{1}{2} Q^{2}\left\langle u(z)^{2}\right\rangle\right)$

where $Q$ is the magnitude of the scattering vector $Q=4 \pi \sin \theta / \lambda$ ( $\theta$ and $\lambda$ being the diffraction angle and the X-ray wavelength, respectively). A perfect (defect-free) crystal is characterized by a $\mathrm{DW}=1$, whereas a highly defective, or an amorphous material, will have a $\mathrm{DW}=0$. It has to be noted that in the case of displacement cascades, very large displacements from the average atomic position can be encountered, so that the finite variance condition might be unjustified. Removing this condition leads to the so-called "generalized central limit theorem" which states that the sum of identically distributed random variables converges to a Lévy-stable distribution. Considering a symmetrical Lévy-stable distribution, the DW factor can be written:

$$
D W(z)=\exp \left(-\frac{1}{2} \mid Q^{\gamma} \sigma_{u}^{\gamma}\right)
$$

where $\gamma$ is the tail index, that determines the shape of the displacement distribution function, and $\sigma_{u}$ is the "characteristic width" of the distribution [11]. It can be noted that $\gamma=2$ corresponds to a Gaussian, and $\gamma=1$ to a Lorentzian distribution. It should be mentioned that thermal vibrations also contribute to lower the diffracted amplitude through a term given by equation (2) (except that the average $<_{\ldots} . .>$ is a time average). In order to disentangle both contribution, the Bragg peak of the unirradiated part of the crystal is used as an intensity reference, so that in the following simulations the sole static disorder is refined.

For the sake of calculating the diffracted intensity, functional forms have to be assumed for both $e(z)$ and $D W(z)$. The corresponding equations have to be versatile enough to describe the shape of the actual profiles encountered in ion-irradiated crystals while the number of parameters entering the equations has to be as low as possible in order to ease the fitting step. In order to meet these contradictory requirements we have chosen to model $e(z)$ and $D W(z)$ with cubic $B$-spline functions. These functions are written [10]:

$$
f(z)=\sum_{i=1}^{N} w_{i} B_{i, 3}(z)
$$

where $B_{\mathrm{i}, 3}(z)$ is the $i^{\text {th }}$ basis function of order $3, w_{i}$ is the weight given to the basis function and $N$ is the total number of basis functions used to describe the function. The basis functions are bellshaped (with a shape close to Gaussian function) and the degree of details that can be rendered by the $B$-spline function is entirely determined by the number basis function used: a low number of basis function will yield a smooth shape but with the risk of wiping out small details, whereas a high number of basis functions increases the ability to render tiny features but also increases the possibility for $f(z)$ to exhibit unphysical oscillations. In this work we used 7 basis functions for both $e(z)$ and $D W(z)$, which yields 14 fitting parameters.

The diffracted intensity is calculated within the framework of the dynamical theory of diffraction as described by the Takagi-Taupin equations [12]. These equations describe the evolution as a function of depth in the form of differential equation of the ratio of the diffracted and incident amplitude, and are explicit functions of the structural parameters of the crystal (composition, structure, strain and random disorder). The Takagi-Taupin equations were solved using the recursion formula given by Bartels et al. [13]. For this purpose, the irradiated region of the crystal is divided into slices in which the structural parameters are assumed to be constant but vary from 
one slice to the other according to Eqs. (1-4). In the present study, it turned out that 50 slices were sufficient to accurately compute the diffracted intensity. It should be mentioned that within this approach, no information can be obtained regarding the exact nature and density of the defects induced by irradiation. The defect structure is solely described with the two phenomenological parameters introduced above, namely the strain and the damage profiles.

Finally, the calculated intensity is compared to the observed data, and the shapes of $e(z)$ and $D W(z)$ are refined (by varying the weights $w_{i}$ ) until a perfect agreement is reached. This fitting stage is performed by making use of a generalized simulated annealing (GSA) algorithm. This algorithm, contrarily to common local optimization algorithms (such as the steepest descent, simplex or Newton's algorithm, for instance) is able to determine the global minimum of the fitting problem, thereby avoiding unphysical solutions. Moreover, contrary to classical simulated annealing, GSA has been proven to increase the convergence speed by about 2 orders of magnitude [8]. The procedure outlined above has been applied to the determination of the strain and damage profiles in YSZ crystal irradiated with $4 \mathrm{MeV} \mathrm{Au}^{2+}$ ions at different temperatures. The results are discussed below.

\section{Results and discussion}

Fig. 1-3 displays $\theta-2 \theta$ scans recorded in the vicinity of the (400) reflection for virgin and irradiated YSZ crystals at RT (Fig. 1), $500^{\circ} \mathrm{C}$ (Fig. 2) and $800^{\circ} \mathrm{C}$ (Fig. 3). The XRD curves for all the fluences exhibit an intense narrow peak on the high-angle side which corresponds to the unirradiated part of the samples. This was expected since the thickness probed by X-rays in this configuration ( $4 \mu \mathrm{m}$ with the $1 / e$ attenuation-length criterion) is greater than the damaged thickness $(\sim 1 \mu \mathrm{m})$. The position of this peak will be used to determine the strain free lattice parameter of YSZ $\left(a_{\mathrm{YSZ}}\right.$ in Eq. 1$)$.

For the irradiated samples, we observe an additional signal on the low-angle side (i.e. in the region of higher lattice parameter) which originates from the damaged part of the crystal. This clearly indicates that the irradiated region is tensily strained in the direction normal to the surface. Moreover, the asymmetrical intensity distribution in this region indicates the existence of a dilatation gradient. It has been shown that the formation of interstitial defects during irradiation might be at the origin of this lattice expansion [3]. Upon increasing fluence, the peak from the damaged region moves towards lower angles, indicating an increase of the tensile strain and hence an increase of the defect density. For the highest fluences, the intensity of the signal from the irradiated region dramatically decreases indicating a strong increase of random atomic displacements. This behavior might be ascribed to a change in the response of YSZ. In particular, in the case of YSZ crystals irradiated at RT, it has been shown that above a critical fluence, point defect clusters re-arrange to form extended defects such as dislocation loops [3, 4], which strongly distort the crystal lattice. The same mechanism occurs at higher temperatures [5]

The simulations of XRD data are also shown in Figs. 1-3 (solid lines). It can be noticed that for all except the last fluences, the simulations are close to perfect and the tiniest details of the fringe structure of the diffraction profiles are very well rendered (especially at high fluences). This allows us to claim that the strain and damage profiles obtained from the simulations are most likely very close the actual state of strain and disorder in the crystals and can hence be analyzed 
confidently. For the highest fluences $\left(5 \times 10^{15}\right.$ for RT and $500^{\circ} \mathrm{C} ; 10^{15} \mathrm{~cm}^{-2}$ for $\left.800^{\circ} \mathrm{C}\right)$, the agreement is less satisfactory, which mainly stems from the fact that the intensity emanating from the irradiated region is extremely weak and featureless, which significantly impairs the fitting. The strain and DW profiles obtained from the simulations are shown in Fig. 4-6. Since the statistical properties of the atomic displacements within the displacement cascades induced by irradiation is not precisely known, we shall not discuss the $\left\langle u(z)>\left(\right.\right.$ or $\left.\sigma_{u}\right)$ and restricted ourselves to the analysis of the DW term.

Let us first discuss the strain profile (Fig. 4-6a). Upon increasing fluence, for all temperatures, it can be observed that the maximum strain level in the irradiated region increases, in perfect agreement with the qualitative observation of the XRD data. In addition it can also be observed that the width of the damaged region increases and globally the depth of the maximum strain is shifted towards higher values. For the two last fluences and the two highest temperatures, in a selected range of depth, the strain profiles exhibit an unrealistic oscillating shape (shown as dotted lines). This behavior can be understood by the examination of the DW profiles displayed in Fig. 5-6b. Upon increasing fluence, as expected from the qualitative observation of the curves, we clearly observe an increase in the level of disorder (decrease of DW). Similarly to what is observed for the strain, the width of the damaged region increases with increasing fluence. Interestingly, for the highest fluence, the DW is close to 0 (say $<0.05$ ) over a wide range of depth $(\sim 1 \mu \mathrm{m})$. This finding indicates that the YSZ crystal in these regions is so defective that its diffracted Bragg intensity is close to 0, i.e. almost no X-rays are emitted from these regions in the symmetrical $\theta-2 \theta$ configuration. As a consequence, no physical strain value related to these regions can be obtained by $\mathrm{XRD}$ in this configuration. This simple observation explains the occurrence of an unphysical behavior of the strain in these regions. The observed oscillations are due to the fact that the strain profile is bounded by the B-spline model that prevents the strain to diverge or exhibit abrupt variations during the fitting procedure. Conversely, this behavior is not observed at RT (Fig. 4). This can be explained by the fact that the width of the heavily damaged region is much smaller $(\sim 200 \mathrm{~nm})$ than for the highest temperature.

Fig. 7 displays the evolution of the maximum strain and damage as a function of the ion fluence. These figures are obtained by selecting the maximum physical strain and the minimum DW values in the profiles presented above. As reported previously [3, 4, 5], both kinetics exhibit a two-step behavior.

Let us first examine the evolution of damage. We clearly observe a slow increase in damage up to a critical fluence, followed by a dramatic increase beyond this fluence, similar to what has been observed using Rutherford backscattering spectroscopy in back channeling mode (RBS/C). However the absolute values of the damage are markedly different: a damage equal to 1 ( $\mathrm{DW}=$ 0 ) is obtained by XRD, whereas the damage fraction obtained by RBS/C is always lower than 1 . This difference can be ascribed to differences in the sensitivity of both techniques. XRD results from the constructive interferences between the waves scattered by all atoms and is hence very sensitive to minute atomic displacements. On the other hand, it is expected that much larger displacements are required to induce backscattering/dechanneling in RBS. It should also be borne in mind that XRD in the symmetric $\theta-2 \theta$ configuration probes out-of-plane displacements, whereas RBS/C performed normal to the surface probes in-plane lattice displacements. Therefore, anisotropic strain fields, such as that observed in irradiated single crystals[14], might induce 
different responses depending on the direction analyzed.

In addition, two other interesting features should be noted. First, below the threshold fluence, increasing the temperature results in a partial but noticeable healing of the material (i.e. the damage is less pronounced) which can be ascribed to an enhanced annihilation rate of defects (mainly self interstitials) as a result of an enhanced mobility (the same conclusion holds for the strain). Second, the transition fluence is shifted towards lower values at higher temperatures (from $10^{15}$ to $2-4 \times 10^{14} \mathrm{~cm}^{-2}$ ). As proposed in [15], above a critical size, point defect clusters rearrange to form extended defects such as dislocation loops. An enhanced defect-clustering rate due to a larger mobility with increasing temperature could be invoked to explain the shift in the transition fluence observed here.

Let us now analyze the evolution of strain. Here also, we observe a two-step mechanism (with a lower strain at higher temperatures, as discussed above). In Figs. 4-6 we plotted the value of the strain measured in the region where the DW factor is not equal to 0 (full lines). In the heavily damaged regions (DW < 0.05), a strain relaxation involving plastic deformation takes place [4]. Thus, whilst the damage level increases, the elastic strain is partially relieved. With the present simulations, it is possible to retrieve the elastic strain in regions of crystals where plastic relaxation did not yet occur, generally at the damaged/pristine interfaces. Figs. 5-6 show that the strain continues to increase in these regions up to $0.3 \%$ and $0.5 \%$ at $500^{\circ} \mathrm{C}$ and $800^{\circ} \mathrm{C}$, respectively, i.e., values clearly lower than the one measured at RT $(0.9 \%)$ before plastic relaxation. These results shows that at higher temperatures, the plastic relaxation mechanism is triggered at lower strain values which is consistent with an enhanced defect mobility.

\section{Conclusion}

Strain and damage profiles in YSZ single crystals irradiated with $4 \mathrm{MeV} \mathrm{Au}^{2+}$ ions at different temperatures, have been studied by means of X-ray diffraction. Although in principle, XRD is highly sensitive to atomic displacements, the determination of displacement profiles is hindered by the phase problem. We have shown that this issue can be circumvented by a fitting approach where the strain and damage profiles are modeled with cubic $B$-spline functions, and the diffracted intensity is computed using the dynamical theory of diffraction. The fitting itself is performed using the generalized simulated annealing algorithm that allows to find the global minimum of the fitting problem. With this approach, the complete strain and damage profiles could be retrieved. Upon increasing fluence, the width of the damaged region increases and both the level of strain and damage inside this region increases. For the highest fluences the damage level is so high that the strain cannot be measured anymore in the most defective regions but can still be obtained at the damaged/pristine interfaces. The damage build-ups obtained at different temperatures revealed the same two-step mechanism for the behavior of YSZ upon ion irradiation. Increasing the temperature induces an enhanced defect clustering, that both lowers the strain and damage in the first step of the damage build-up and shifts the threshold fluence towards lower values.

\section{Acknowledgements}

XRD measurements on the PANalytical diffractometer have been performed at the nanocenter CTU-IEF-Minerve that is partially funded by the "Conseil Général de l'Essonne". We thank the NEEDS-Matériaux CNRS program for its partial funding. J. Channagiri is thankful to the région 
Limousin for the partial funding of this project. All calculation have been performed using the python programming language (http://www.python.org/) combined with the Numpy (http://www.numpy.org/), Scipy (http://www.scipy.org/) and MatPlotLib (http://matplotlib.org/) libraries. The corresponding developers and communities are gratefully acknowledged. 


\section{References}

1. W. J. Weber, R.C. Ewing, C.R.A. Catlow, T.D. dela Rubia, L.W. Hobbs, C. Kinoshita, H. Matzke, A.T. Motta, M. Nastasi, E.K.H. Salje, E.R. Vance, and S.J. Zinkle, J.Mater. Res., 13 (1998) 1434

2. K. E. Sickafus, H. Matzke, Th. Hartmann, K. Yasuda, J.A. Valdez, P. Chodak III, M. Nastasi, R.A. Verrall, J. Nuc. Mater., 274(1) (1999) 66

3. S. Moll, L. Thomé, G. Sattonnay, A. Debelle, F. Garrido, L. Vincent, J. Jagielski, J. Appl. Phys. 106 (2009) 073509

4. A. Debelle, S. Moll, B. Décamps, A. Declémy, L. Thomé, G. Sattonnay, F. Garrido, I. Jozwik, J. Jagielski, Scr. Mater. 63 (2010) 665

5. A. Debelle, L. Thomé, A. Boulle, S. Moll, F. Garrido, L. Qasim, P. Rosza, Nuc. Instr. Meth. B. 277 (2012) 14

6. J. Jagielski, L. Thomé, Appl. Phys. A 97(1) (2009) 147.

7. A. Debelle, F. Garrido, L. Thomé, Mater. Res. Soc. Symp. Proc. Vol. 1265 (2010) 1265AA08-07-BB07-07.

8. A. Boulle, A. Debelle, J. Appl. Cryst. 43 (2010) 1046

9. Special issue of Acta Cryst. A 69, Part 1, January 2013

10. A. Boulle, O. Masson, R. Guinebretière, A. Dauger, J. Appl. Cryst. 36(6) (2003) 1424

11. A. Boulle, R. Guinebretière, A. Dauger, J. Phys. D: Appl. Phys. 38 (2005) 3907

12. A. Authier, Dynamical Theory of X-ray Diffraction, $\mathrm{IUCr}$ Monographs on Crystallography 11, Oxford University Press (2005)

13. W. J. Bartels, J. Hornstra, \& D.J.W. Lobeek, Acta Cryst. A 42 (1986) 539

14. A. Debelle and A. Declémy, Nucl. Instr. Meth. B, 268 (2010) 1460

15. A.I. Ryazanov, K. Yasuda, C. Kinoshita, A.V. Klaptsov, J. Nucl. Mater. 307-311 (2002) 918. 


\section{List of Figure Captions}

Fig 1. $\theta-2 \theta$ scans (dark circles) and corresponding simulated curves(colored lines) recorded in the vicinity of the $\left(\begin{array}{lll}4 & 0 & 0\end{array}\right)$ reflection for virgin and YSZ crystals irradiated at room temperature for increasing Au-ion fluences (expressed in $\mathrm{cm}^{-2}$ ). The intensities are shifted vertically for clarity.

Fig 2. $\theta-2 \theta$ scans (dark circles) and corresponding simulated curves(colored lines) recorded in the vicinity of the $\left(\begin{array}{lll}4 & 0 & 0\end{array}\right)$ reflection for virgin and YSZ crystals irradiated at $500^{\circ} \mathrm{C}$ for increasing $\mathrm{Au}-$ ion fluences (expressed in $\mathrm{cm}^{-2}$ ). The intensities are shifted vertically for clarity.

Fig 3. $\theta-2 \theta$ scans (dark circles) and corresponding simulated curves(colored lines) recorded in the vicinity of the $\left(\begin{array}{lll}4 & 0 & 0\end{array}\right)$ reflection for virgin and $\mathrm{YSZ}$ crystals irradiated at $800^{\circ} \mathrm{C}$ for increasing $\mathrm{Au}-$ ion fluences (expressed in $\mathrm{cm}^{-2}$ ). The intensities are shifted vertically for clarity.

Fig 4. Strain (a) and Debye-Waller (b) depth profiles obtained from the fitting of XRD curves displayed in Fig. 1 (room temperature).

Fig 5. Strain (a) and Debye-Waller (b) depth profiles obtained from the fitting of XRD curves displayed in Fig. $2\left(500^{\circ} \mathrm{C}\right)$.

Fig 6. Strain (a) and Debye-Waller (b) depth profiles obtained from the fitting of XRD curves displayed in Fig. $3\left(800^{\circ} \mathrm{C}\right)$.

Fig 7. Variation of the maximum strain and damage levels as a function of the ion fluence. 


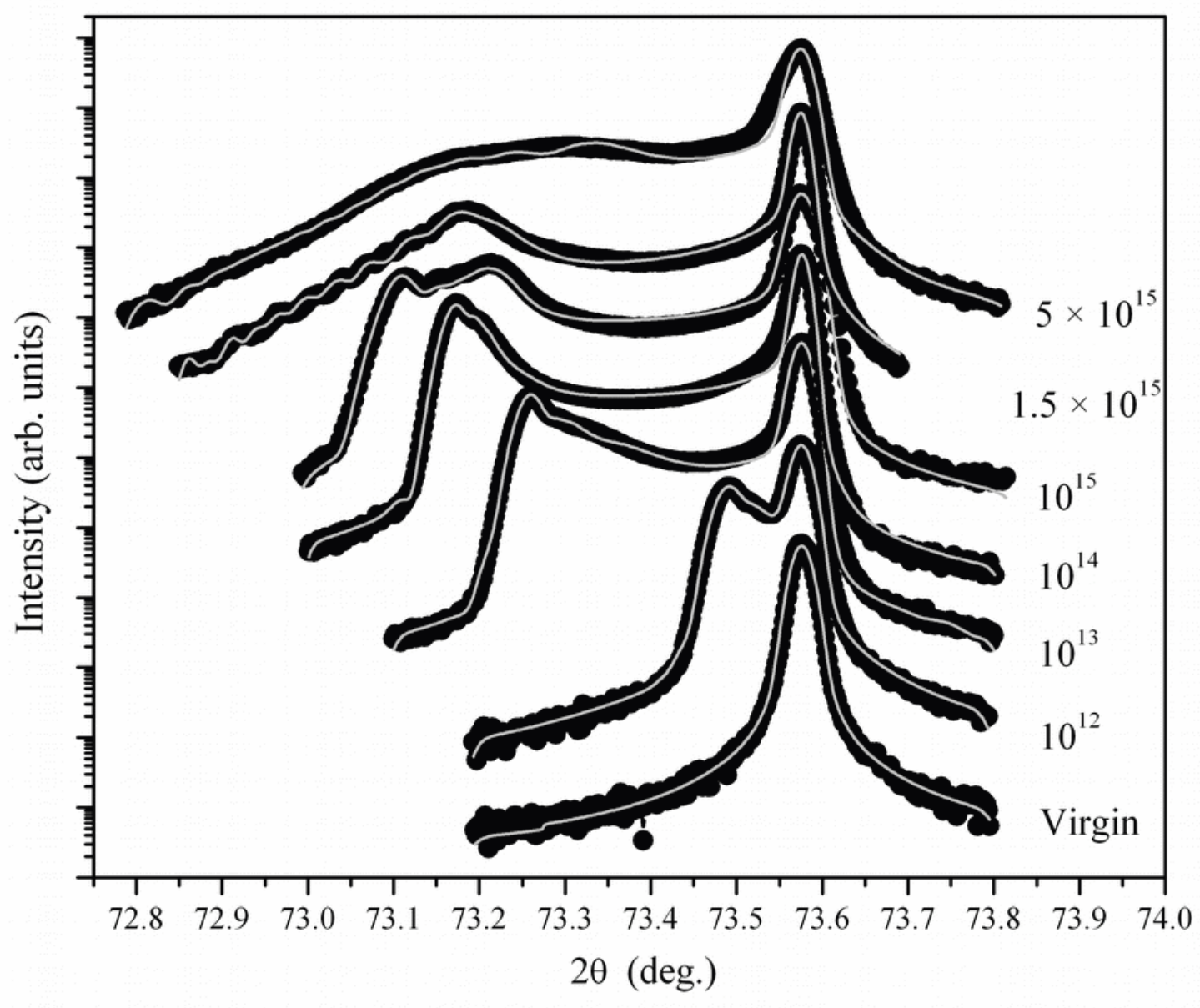




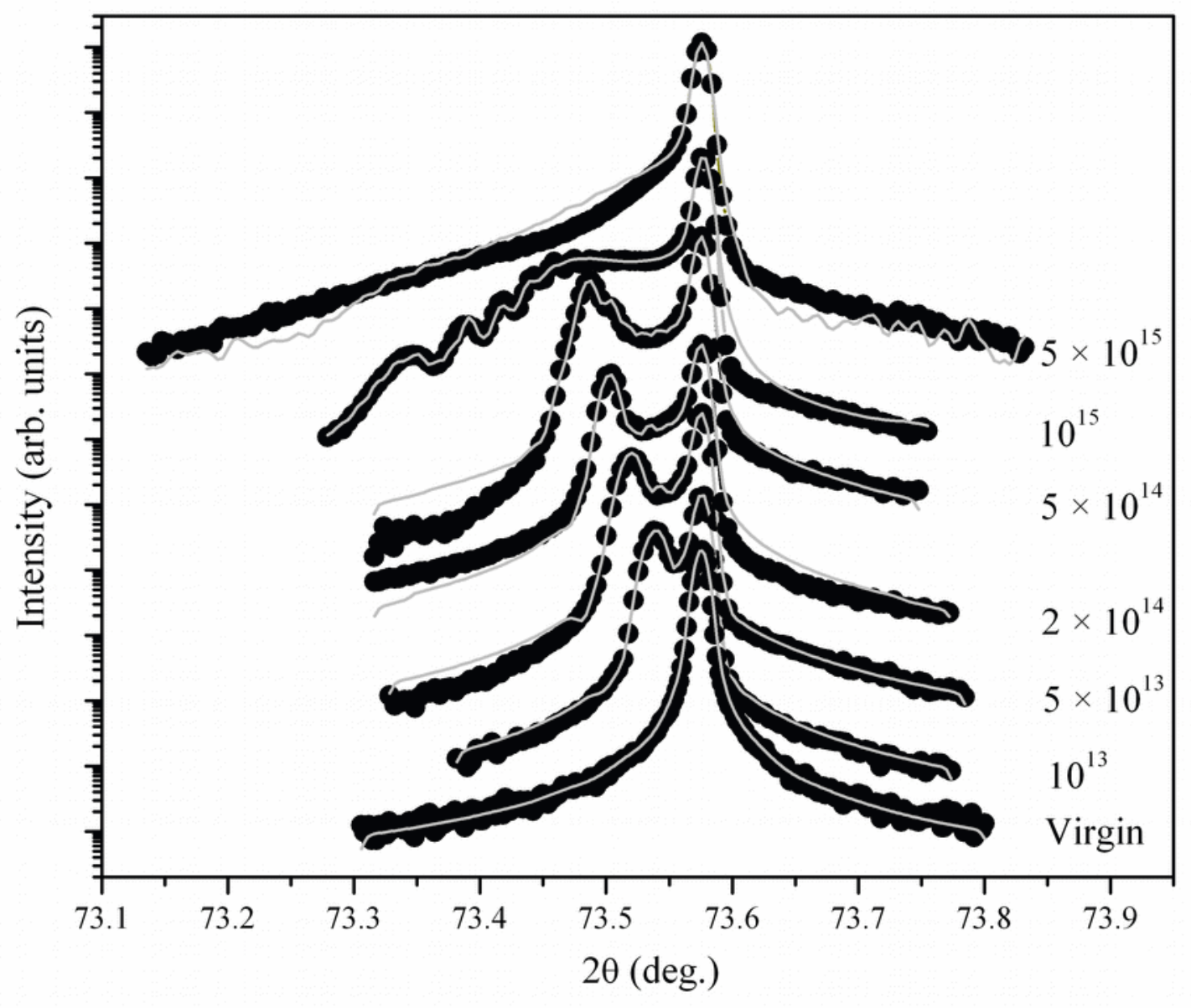




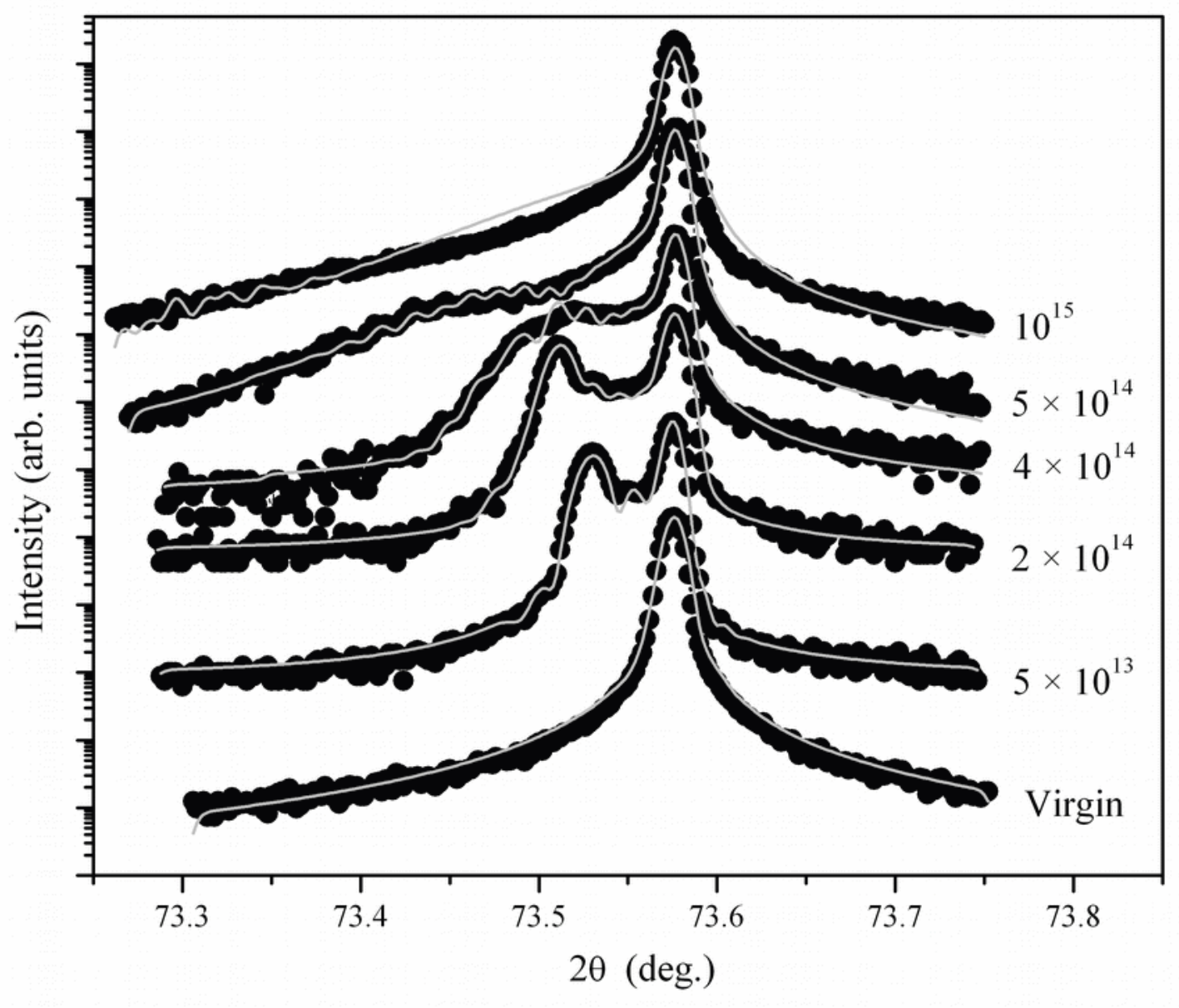




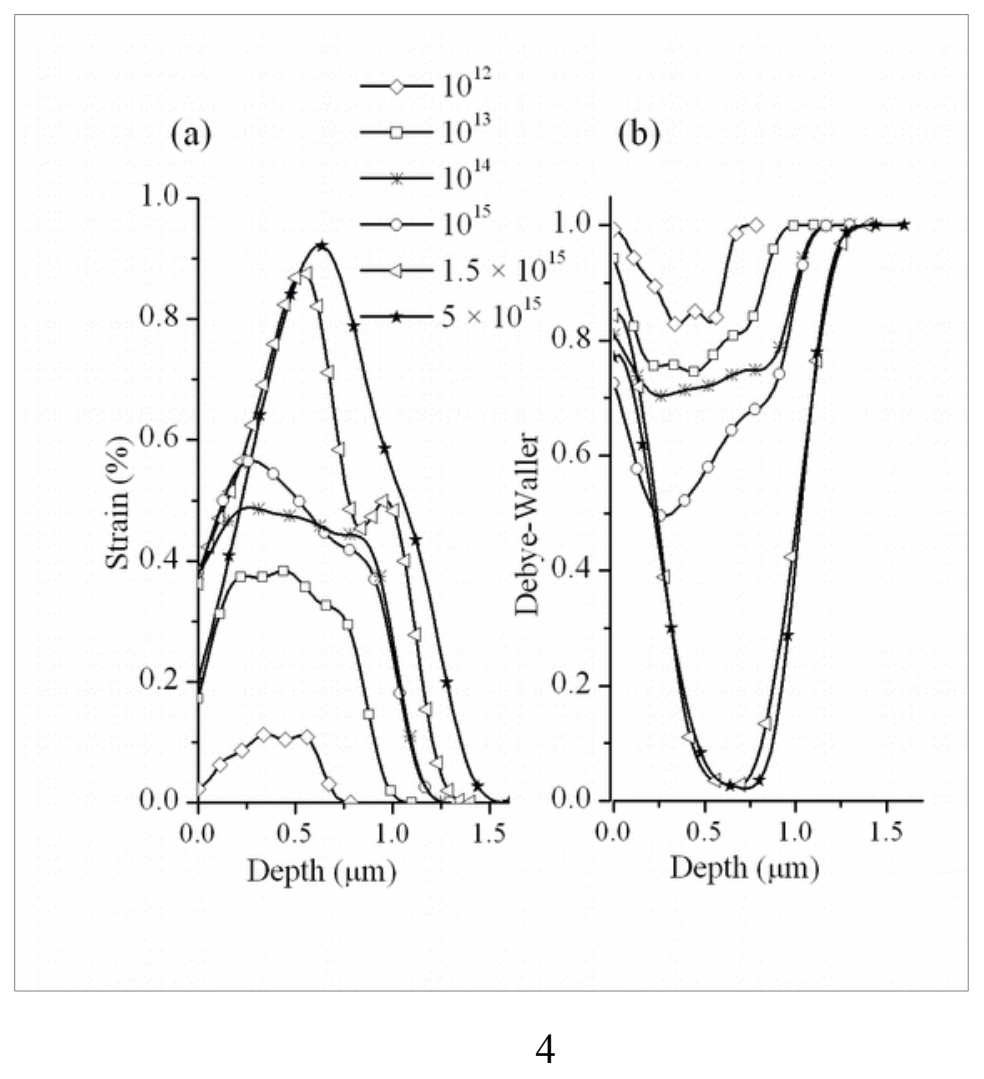




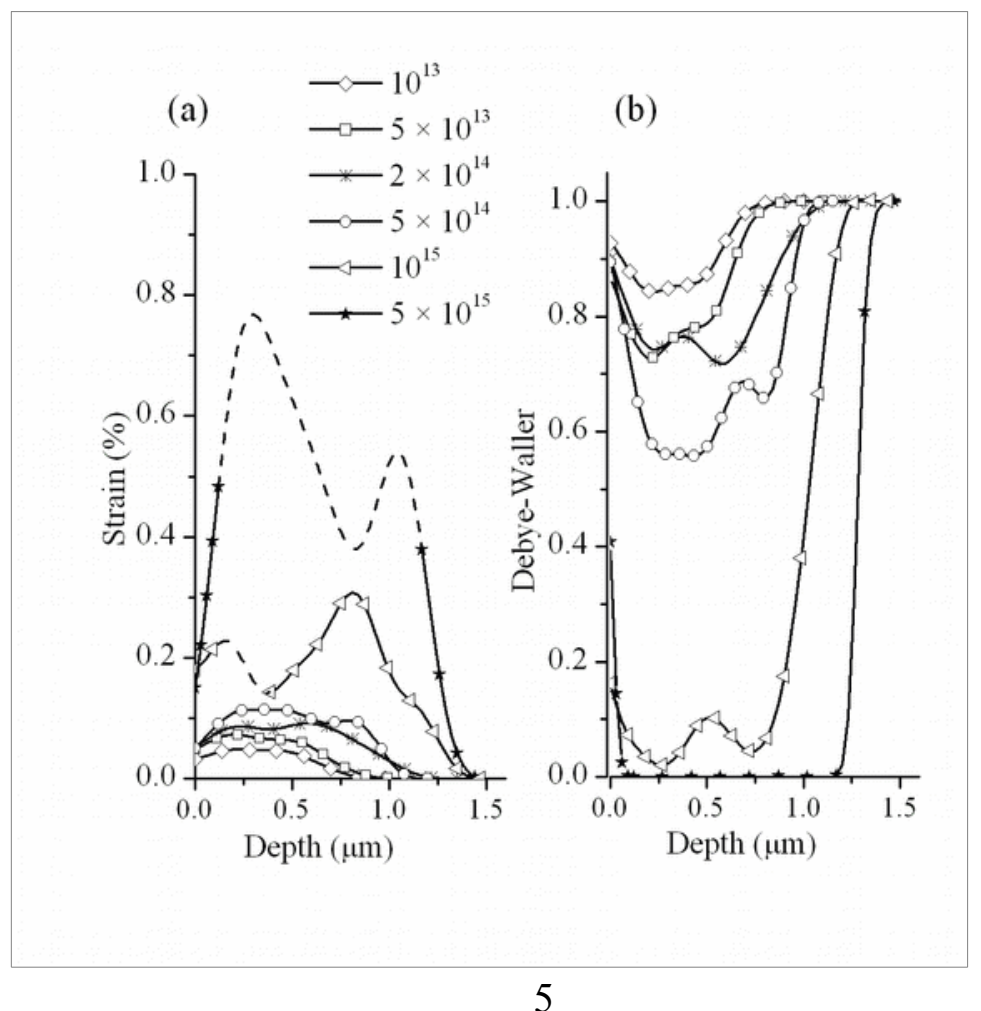




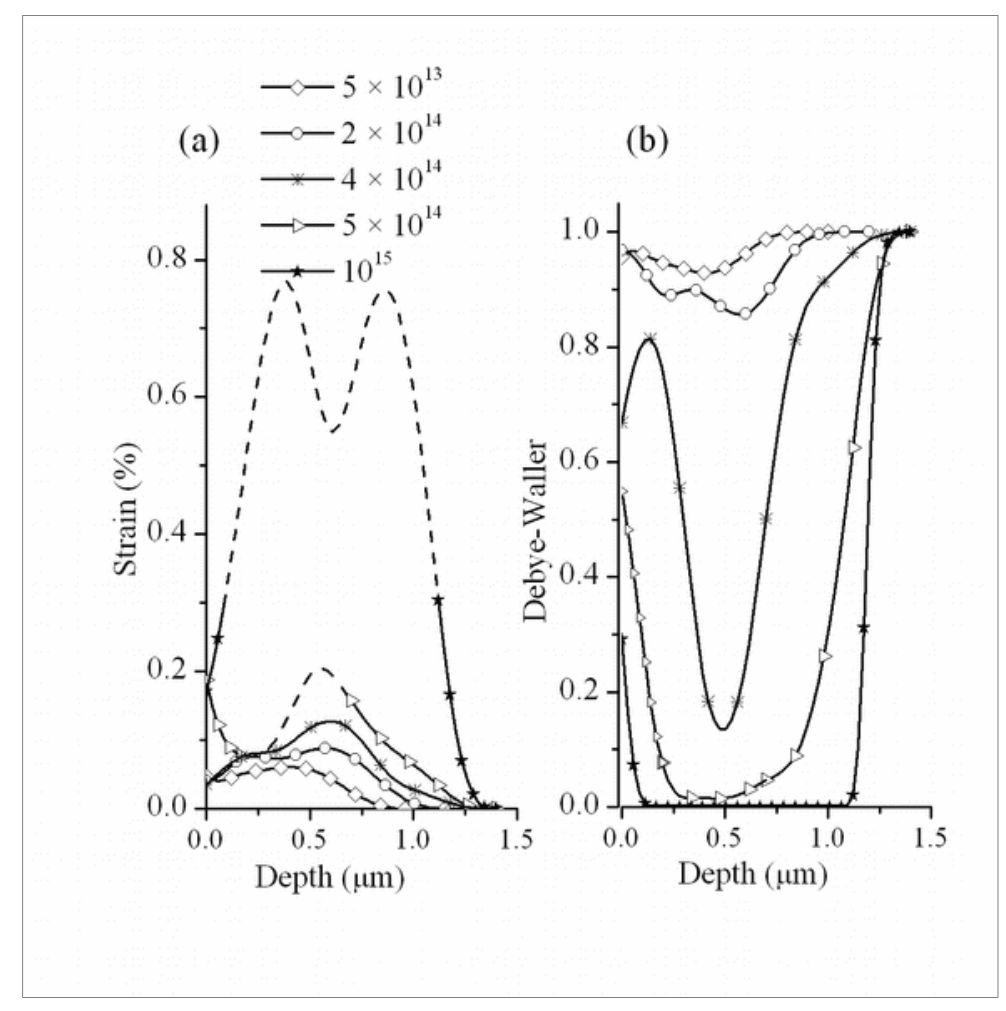




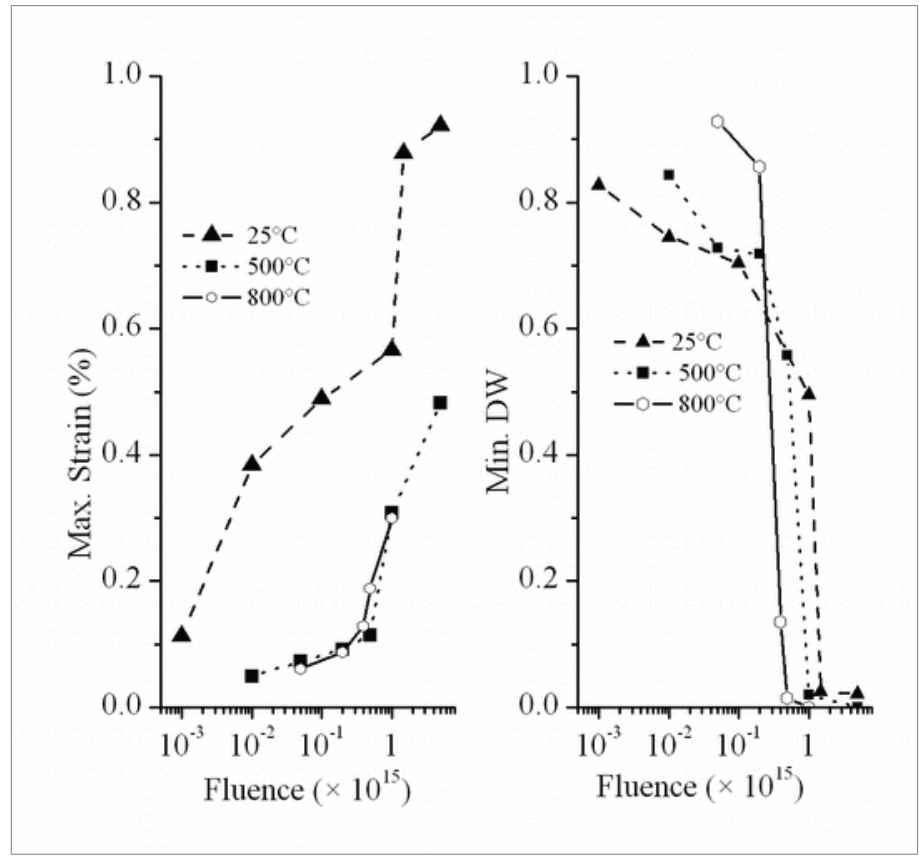

